Artículo

\title{
Instalación y evaluación de secador solar autónomo para secado de papa en Tarma
}

\author{
Bécquer Frauberth Camayo Lapa ${ }^{18}$ \\ Miguel Ángel Quispe Solano ${ }^{2}$ \\ Alex Rubén Huamán De La Cruz ${ }^{3}$ \\ David Elvis Condezo Hurtado ${ }^{4}$ \\ Juan Raúl Massipe Hernández ${ }^{5}$ \\ Liz Evelyn Landa Guadalupe ${ }^{1}$
}

${ }^{1}$ Facultad de Ciencias Aplicadas-Universidad Nacional del Centro del Perú. Av. Mariscal Castilla núm. 3909, El Tambo, Huancayo, Perú (lizlanda94@hotmail.com). ${ }^{2}$ Facultad de Industrias AlimentariasUniversidad Nacional del Centro del Perú. Av. Mariscal Castilla Núm 3909, El Tambo, Huancayo, Perú (quispe_miguelangel@hotmail.com). ${ }^{3}$ Instituto General de Investigación-Universidad Nacional del Centro del Perú, Av. Mariscal Castilla Núm. 3909, El Tambo, Huancayo, Perú (alebut2@ hotmail.com). ${ }^{4}$ Maestría en Gestión del Mantenimiento de Sistemas Energéticos-Facultad de Ingeniería eléctrica y ElectrónicaUniversidad Nacional del Centro del Perú. Av. Mariscal Castilla Núm. 3909, El Tambo, Huancayo (davidelvis7@ hotmail.com). ${ }^{5}$ Doctorado en Máquinas y Motores Térmicos. AlmaRed Global-España. Calle de los Alamillos núm. 28260, Galapagar, Madrid. (raulm026@ hotmail.com).

§Autor para correspondencia: camayobecquer@hotmail.com.

\section{Resumen}

En este trabajo se ha desarrollado un secador solar autónomo y automatizado para secar papa en la localidad de Tarma a una altitud de 3040 m en julio de 2019, el cual cuenta con un colector solar con dos reflectores, cámara de secado con capacidad de $20 \mathrm{~kg}$, panel fotovoltaico y un sistema de control. Se estudió el comportamiento térmico del secador solar sin carga, logrando incrementos de la temperatura en la cámara de secado de hasta $50.7 \pm 7{ }^{\circ} \mathrm{C}$ con radiaciones solares de $880 \pm 144$ $\mathrm{W} \mathrm{m} \mathrm{m}^{-2} \mathrm{~h}^{-1}$. Se evaluó el efecto del pretratamiento con dos concentraciones de bisulfito de sodio $(0.02 \%$ y $0.03 \%)$ y tres temperaturas de secado $\left(50{ }^{\circ} \mathrm{C}, 55{ }^{\circ} \mathrm{C}\right.$ y $\left.60{ }^{\circ} \mathrm{C}\right)$ para evaluar las características físicas $\left(\mathrm{L}^{*}\right)$, fisicoquímicas (acidez, $\mathrm{pH}$ ) y químicas (humedad y cenizas). Los resultados mostraron una reducción de $70 \%$ del agua teniendo una humedad final entre $12.56 \%$ a $13.85 \%$. Asimismo, no fue encontrado diferencia significativa $(p>0.05)$ entre las características físicas, fisicoquímicas y química a las diferentes concentraciones de bisulfito y temperaturas de secado de la papa Yungay. Estos parámetros de calidad de papa se encuentran de acorde a la norma peruana NTP 011.119 'papa y sus derivados: papa, definiciones y requisitos', lo que ofrece una alternativa eficiente al deshidratado de productos agrícolas.

Palabras claves: automatizado, papa, secador solar autónomo, secado solar indirecto.

Recibido: mayo de 2020

Aceptado: julio de 2020 


\section{Introducción}

A nivel mundial, la mayoría de los agricultores buscan reducir el contenido de humedad de sus cultivos cosechados, esto con el objetivo de evitar su deterioro durante su almacenamiento. El secado es el proceso de remoción de la humedad a través de un simultaneo calentamiento y transferencia de masa (El-Sebaii y Shalaby, 2012). El secado es uno de los métodos más antiguos, clásicos y comúnmente utilizado para preservar y extender la vida útil de productos agrícolas tales como granos, verduras, frutas y otros productos (Chemkhi et al., 2004).

En países en desarrollo como Perú, su aplicación muchas veces está restringida a secadores convencionales (dejar el producto sin control ninguno en contacto con el sol y contaminantes) o procesos industriales donde usualmente se consumen enormes cantidades de energía eléctrica (López y Chávez, 2018). Por lo tanto, el uso de energía solar como secado térmico donde se pueda tener control de variables como temperatura y humedad (para mejorar la calidad del producto) y a la vez reducir el impacto económico y ambiental sería de gran valía (Bergues et al., 2008).

Por ejemplo, Rodríguez-Tapia et al. (2017), instalaron, automatizaron, y evaluaron un secador solar, reportando una disminución en el tiempo de secado (entre 30\% y 50\%), reducción en los costos, reducción de pérdidas por ataques de plagas, mejoras significativas en la calidad del producto final, y control y reproducibilidad del proceso. Asimismo, Prada et al. (2019) reportaron reducción en la humedad del grano de café hasta $12.3 \%$ en solo cinco días y Quintanar et al. (2019) evaluaron la inversión económica y su rentabilidad de un secador solar tipo invernadero para madera, consiguiendo una rentabilidad de hasta $240 \%$ de su inversión.

Perú es líder en producción de papa en Latinoamérica (décimo cuarto en el mundo) y cuenta con más de 3000 variedades de papas, sembradas en cerca de 70000 ha; sin embargo, solamente 12\% es comercializado a nivel local y el resto muchas veces son deteriorados en las áreas de cultivo o su distribución (Agraria, 2019). Por lo tanto, un adecuado tratamiento para su conservación es necesario. La calidad de frutas y vegetales durante el secado usando secador solar ha sido estudiado por varios investigadores. Por ejemplo, Iglesias et al. (2017), testaron la calidad del secado solar sobre el mango ataulfo en Chiapas-México, encontrando valores de $5 \%, 45^{\circ} \mathrm{C}$ y $25^{\circ} \mathrm{C}$ referentes a la humedad y temperatura de la cámara de secado y temperatura ambiente, respectivamente.

El tiempo de secado fue de 8 horas con $500 \mathrm{~W} \mathrm{~m}^{-2}$ de radiación solar y con reducción de la humedad de $80 \%$ (inicial) hasta $8.4 \%$ (final). Lati et al. (2017) evaluaron el efecto del secador solar sobre la calidad de la papa; a través, del análisis de color, reducción del azúcar y pH, bajo un tratamiento controlado de $50{ }^{\circ} \mathrm{C}$. Por su parte, Chouicha et al. (2013) estudiaron el secador híbrido solar sobre papa en rodajas y encontraron un tiempo de secado de $3 \mathrm{~h}$ y contenido de humedad final de $0.13 \%$. Asimismo, Tefera et al. (2013) utilizando un secador solar encontró una reducción en peso de 0.9 $\mathrm{kg}$ a alrededor de $0.19 \mathrm{~kg}$ en dos días, reduciendo entre $2 \mathrm{~h}$ a $3 \mathrm{~h}$ comparado aun día soleado normal.

Por lo tanto, en este trabajo, como una forma de conservación de alimentos que cumpla las normas técnicas de calidad NTP.011.110:2010 (Indecopi, 2010) para el mercado nacional del Perú, se han planteado tres objetivos: i) diseñar y desarrollar un secador solar automatizado para secar productos agrícolas, que abundan en la ciudad de Tarma, como una forma de conservación de estos alimentos; ii) estudiar el comportamiento térmico del secador solar automatizado al vacío y su sistema de 
control de la temperatura en la cámara de secado; y iii) evaluar la viabilidad del secado de papa Yungay en el secador solar autónomo automatizado y el efecto de la temperatura de secado y la solución de bisulfito de sodio sobre la calidad de las papas para su posterior comercialización.

\section{Materiales y métodos}

\section{Preparación de la muestra}

La papa de la especie Yungay fue comprada de los agricultores de Tarma debido a que el sistema de secador solar fue instalado dentro de las instalaciones de la Facultad de Ciencias Aplicadas de la Universidad Nacional del Centro del Perú (UNCP)-filial Tarma, el cual se encuentra localizado a una altitud de $3040 \mathrm{msnm}$ (latitud de $11^{\circ} 25^{\prime} 12^{\prime}$ ' S y longitud de $75^{\circ} 41^{\prime} 17^{\prime \prime} \mathrm{O}$ ). Su clima es árido y semifrío con velocidad promedio del viento de $1.1 \mathrm{~m} \mathrm{~s}^{-1}$, temperatura promedio anual variando entre $13{ }^{\circ} \mathrm{C}$ a $18{ }^{\circ} \mathrm{C}$ y radiación solar media de $5.4 \mathrm{~kW} \mathrm{~h}^{-1} \mathrm{~m}^{-2}$ día ${ }^{-1}$ como reportado por Camayo-Lapa et al. (2017).

Las papas de similar diámetro y tamaño fueron seleccionadas, lavadas y trozadas en cubos de $1 \mathrm{~cm}$ x $1.5 \mathrm{~cm}$. Los trozos entonces fueron esparcidos dentro en un cuarto de secado. En total $850 \mathrm{~g}$ de peso fue utilizado para cada experimento. La humedad relativa inicial medida en las muestras de papa fue de $67 \%$.

\section{Caracterización física, fisicoquímica y químico proximal de la muestra}

Se evaluó el color mediante un colorímetro (Lovibond, RT100, Alemania) con iluminante D65 y observador estándar de $10^{\circ}$ en los que los espectros reflexión de las muestras fueron determinados las coordenadas del CIE- $\mathrm{L}^{*} \mathrm{a}^{*} \mathrm{~b}^{*}$, donde la luminosidad $\left(\mathrm{L}^{*}\right)$, las características fisicoquímicas como la acidez total siguiendo el método titulométrico; 942.15 (AOAC, 1996), el resultado fue expresado como porcentaje de ácido sulfúrico. $\mathrm{El} \mathrm{pH}$ fue determinado por el método potenciométrico, usando un pH metro (Schott, PH11, Alemania) y la composición químico proximal con los métodos recomendados por la Association of Official Agricultural Chemists - AOAC International (AOAC, 2006). En ceniza (método de cenizas totales) y humedad (método de secado por estufa).

\section{Secador solar indirecto autónomo}

En la Figura 1, se observa el colector solar y sus dos reflectores que son espejos planos y en su conjunto, forma el sistema de captación térmico, se encargan de capturar la radiación solar y transformarla en energía térmica (calor) para calentar el aire que pasa a la cámara de secado donde está el producto a secar.

En este estudio la cámara de secado permite secar hasta $20 \mathrm{~kg}$ de producto y cuenta con un sistema de ventilación incorporado, sensores de humedad, peso, ambos monitoreados por medio de un display electrónico acoplado al secador. A través, del display un sistema permite manipular la temperatura en la cámara de secado, de acuerdo con la temperatura de secado requerida, para cada producto según las normas técnicas de calidad. Esta programación de temperatura tiene un rango de $20{ }^{\circ} \mathrm{C}$ a $70{ }^{\circ} \mathrm{C}$ de acuerdo a las recomendaciones de (Infoagro, 2020). 


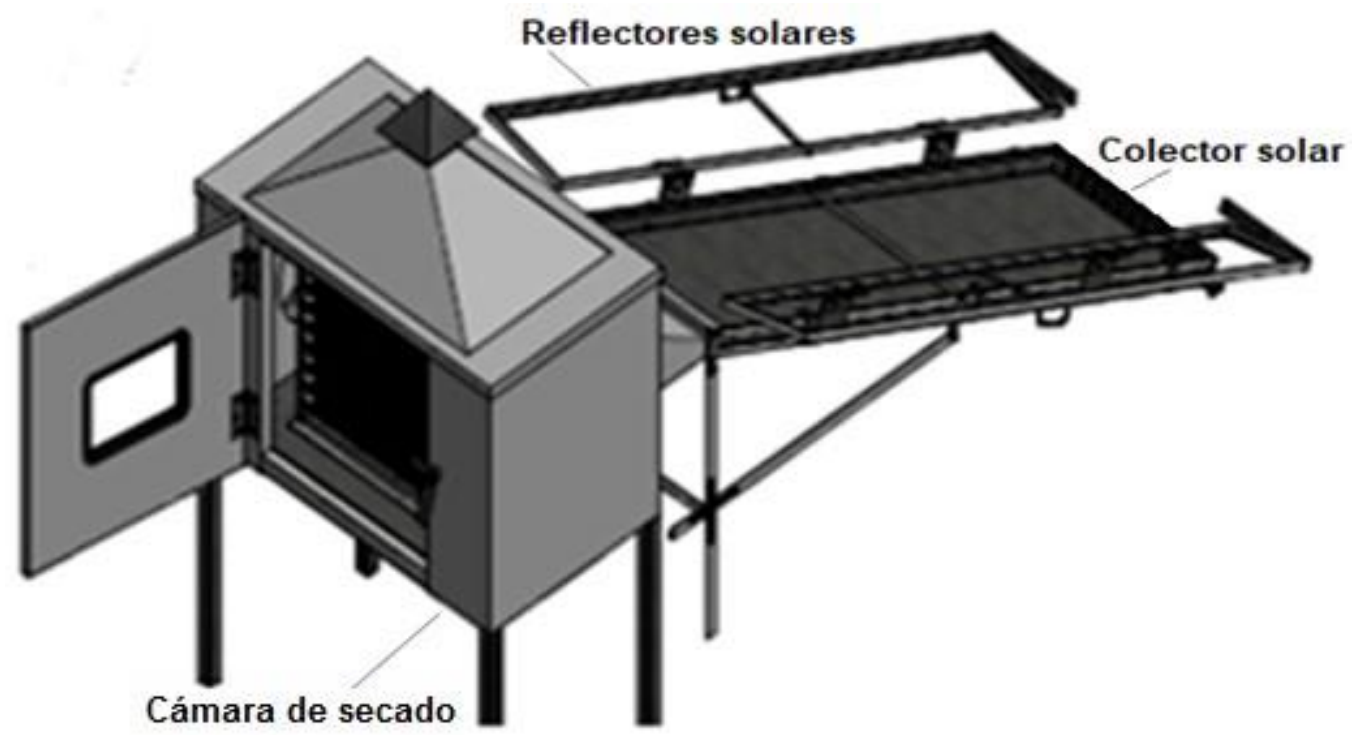

Figura 1. Partes del secador solar indirecto autónomo.

\section{Instalación del secador solar automatizado}

El colector solar y sus reflectores se orientaron al norte geográfico, toda vez que Tarma se encuentra en el hemisferio sur terrestre, de esta manera se aprovecha mejor la radiación solar incidente. En adición, el sistema de captación térmico, orientado al norte, se instaló con una inclinación de 22 grados para favorecer la captación de la energía solar durante los meses de invierno. El colector solar y la cámara de secado fueron conectados a través de un conducto de acero inoxidable de perfil rectangular, el cual evita la fuga y pérdida de aire caliente durante el paso del aire caliente a la cámara de secado.

\section{Diseño, simulación y elaboración del sistema de control}

Para tener control del sistema, una placa del circuito de control fue diseñado e implementado mediante el software Proteus (Versión 8.9, Inglaterra). Además, fueron instalados de sensores en la cabina de secado, en la chimenea y en el colector solar. Se realizaron mediciones del sistema de funcionamiento del secador solar, para ello un algoritmo fue creado con el objetivo de registrar en tiempo real la temperatura y humedad en el display. El diagrama de flujo de las etapas consideradas en el sistema para controlar/manipular la temperatura y humedad es mostrado en la Figura 2.

\section{Contexto para la evaluación de funcionamiento del proceso de secado}

A unos metros de distancia del secador solar, fue instalado una estación meteorológica en donde fueron medidos y registrados variables meteorológicas tales como la temperatura del ambiente $\left({ }^{\circ} \mathrm{C}\right)$, humedad relativa $(\%)$ y la radiación solar $\left(\mathrm{W} \mathrm{m}^{-2} \mathrm{~h}^{-1}\right)$, además de la temperatura interna $\left({ }^{\circ} \mathrm{C}\right)$ de la cámara de secado durante tres días consecutivos entre las 8:00 am a 17:00 pm horario local de la ciudad de Tarma (FACAP-UNCP). 


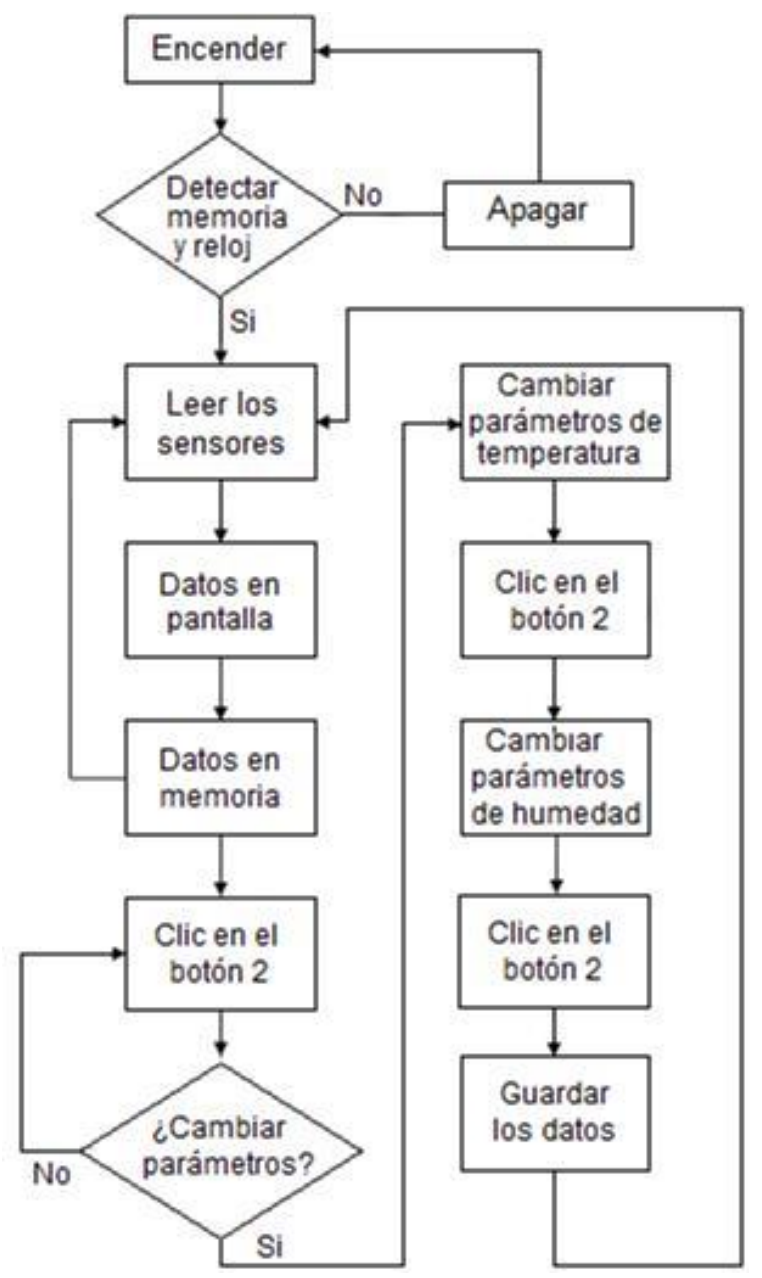

Figura 2. Flujograma del sistema de control de la temperatura y la humedad.

\section{Análisis de datos}

Los datos del análisis físico, fisicoquímico y químico proximal se reportaron como media \pm error estándar, determinados en triplicado. Diferencias entre los tratamientos (temperatura de secado y concentración de sulfito) fueron analizados por análisis de varianza (Anova) de dos vías y posterior Tukey $(p<0.05)$. Las mediciones del comportamiento de radiación solar, temperatura interna y externa en el secador solar fueron graficados utilizando el Software SPSS V.24 y R Project (software libre), versión 3.3.6 (R Team Core, 2019).

\section{Resultados y discusión}

\section{Mediciones del secador solar automatizado sin carga}

Las pruebas preliminares sin carga fueron llevadas a cabo con el objetivo de comprobar el funcionamiento del secador solar. Los resultados promedios \pm desviación estándar (DS) de las mediciones realizadas (temperatura ambiente (Ta), radiación solar (RS), humedad relativa (HR) y temperatura interna del secador (Ti)) sin carga y a cada hora (8:00 h-17:00 h) durante los tres días 
de ensayos, en el secador solar automatizado son mostrados en el Cuadro 1. Los resultados mostraron un rango de temperatura ambiente de $7.3{ }^{\circ} \mathrm{C}$ a $19{ }^{\circ} \mathrm{C}(13: 00 \mathrm{~h})$, mientras que la temperatura interna del secador interno vario entre $10.7^{\circ} \mathrm{C}$ a $50.7^{\circ} \mathrm{C}(12: 00 \mathrm{~h})$.

A su vez, la radiación solar vario entre $72 \mathrm{~W} \mathrm{~m}^{-2} \mathrm{~h}^{-1}$ a $880 \mathrm{~W} \mathrm{~m}^{-2} \mathrm{~h}^{-1}$. Un comportamiento similar fue observado entre las tres variables ( $\mathrm{Ta}, \mathrm{RS}$ y $\mathrm{Ti}$ ) antes mencionados con incremento en su valor de 8:00 h a 13:00 h de la mañana y disminución por el atardecer (14:00 h a 17:00 h), alcanzando sus máximos valores entre las 11:00 h y 14:00 h. En contraste, fue observado disminución de la humedad relativa conforme pasaban las horas, siendo más intenso en los horarios de 9:00 a 12:00 h. Las radiaciones globales y temperatura ambiental registradas por la estación meteorológica Davis en la facultad en Tarma son aproximadamente similares a las estimadas por Camayo-Lapa et al. (2017) de $5.47 \mathrm{~kW} \mathrm{~m}^{-2}$ día $^{-1}$ promedio mensual anual lo que hace que la utilización de cualquier equipo para calentamiento de agua o aire se hace muy rentable. Seveda y Jhajharia (2012) reportaron una reducción de $75 \%$ de la humedad, mostrando resultados similares a este trabajo.

Esto está en concordancia, debido a que mayor energía de radiación solar (13:00 horas mostró la mayor radiación de $880 \pm 144 \mathrm{~W} \mathrm{~m}^{-2} \mathrm{~h}^{-1}$ ) fue capturada durante este periodo. Esto se explica debido a que, si una mayor concentración de radiación solar alcanza un área o superficie, la temperatura de esta área será incrementada (Daut et al., 2012). Estos resultados, mostraron una mayor temperatura interna de la cámara $\left(12: 00 \mathrm{~h}\right.$ mostró la mayor temperatura interna de $\left.50.7 \pm 7{ }^{\circ} \mathrm{C}\right)$ dentro de este periodo, demostrando así, que el sistema del secador solar y el panel fotovoltaico instalados funcionan perfectamente (Cuadro 1).

Cuadro 1. Promedio de la temperatura ambiente, temperatura interna de la cámara de secado, la radiación solar y humedad relativa medidos los días 15, 16 y 17 de julio del 2019.

\begin{tabular}{lcccc}
\hline Hora & $\begin{array}{c}\text { Temperatura ambiente } \\
\left({ }^{\circ} \mathrm{C}\right)\end{array}$ & $\begin{array}{c}\text { Radiación solar } \\
\left(\mathrm{W} \mathrm{m}^{-2} \mathrm{~h}^{-1}\right)\end{array}$ & $\begin{array}{c}\text { Humedad relativa } \\
(\%)\end{array}$ & $\begin{array}{c}\text { Temperatura interna } \\
\left({ }^{\circ} \mathrm{C}\right)\end{array}$ \\
\hline Media $\pm \mathrm{DS}$ & Media $\pm \mathrm{DS}$ & Media $\pm \mathrm{DS}$ & Media $\pm \mathrm{DS}$ \\
\hline $9: 00$ & $7.2 \pm 2.2$ & $72 \pm 26$ & $69 \pm 8$ & $10.7 \pm 2.7$ \\
$10: 00$ & $12.1 \pm 0.8$ & $357 \pm 111$ & $62 \pm 12$ & $24.7 \pm 4.1$ \\
$11: 00$ & $15.0 \pm 1.4$ & $678 \pm 57$ & $42 \pm 17$ & $36.3 \pm 5.5$ \\
$12: 00$ & $17.0 \pm 1.4$ & $723 \pm 227$ & $37 \pm 16$ & $44.7 \pm 1.2$ \\
$13: 00$ & $18.7 \pm 0.9$ & $809 \pm 75$ & $40 \pm 12$ & $50.7 \pm 7$ \\
$14: 00$ & $18.9 \pm 0.9$ & $880 \pm 144$ & $38 \pm 7$ & $41 \pm 7$ \\
$15: 00$ & $18.3 \pm 0.9$ & $677 \pm 136$ & $43 \pm 6$ & $39 \pm 4.9$ \\
$16: 00$ & $16.7 \pm 0.9$ & $509 \pm 150$ & $47 \pm 8$ & $34 \pm 5.4$ \\
$17: 00$ & $15.7 \pm 0.9$ & $281 \pm 135$ & $50 \pm 9$ & $20 \pm 1.7$ \\
\hline
\end{tabular}

DS= desviación estándar.

En la Figura 3, se muestra la temperatura ambiente promedio vs la temperatura promedio interna de la cámara de secado (media de los tres días). La temperatura interna de la cámara de secado mostró un notorio incremento de su temperatura hasta un máximo de $50.7 \pm 7{ }^{\circ} \mathrm{C}$ (esto observado en horario correspondiente a las 12:00 h) debido a la acción del sistema de control que fue configurado a esta temperatura como la máxima. Este resultado corrobora el buen funcionamiento 
del sistema de control, compuesto por dos ventiladores que entran en funcionamiento en caso de que se requiera. Se hace notar que la temperatura interna de la cámara de secado es proporcionar a la temperatura ambiente y la radiación solar ya que mayor temperatura de la cámara más altas temperaturas y valores de radiación solar fueron registrados.

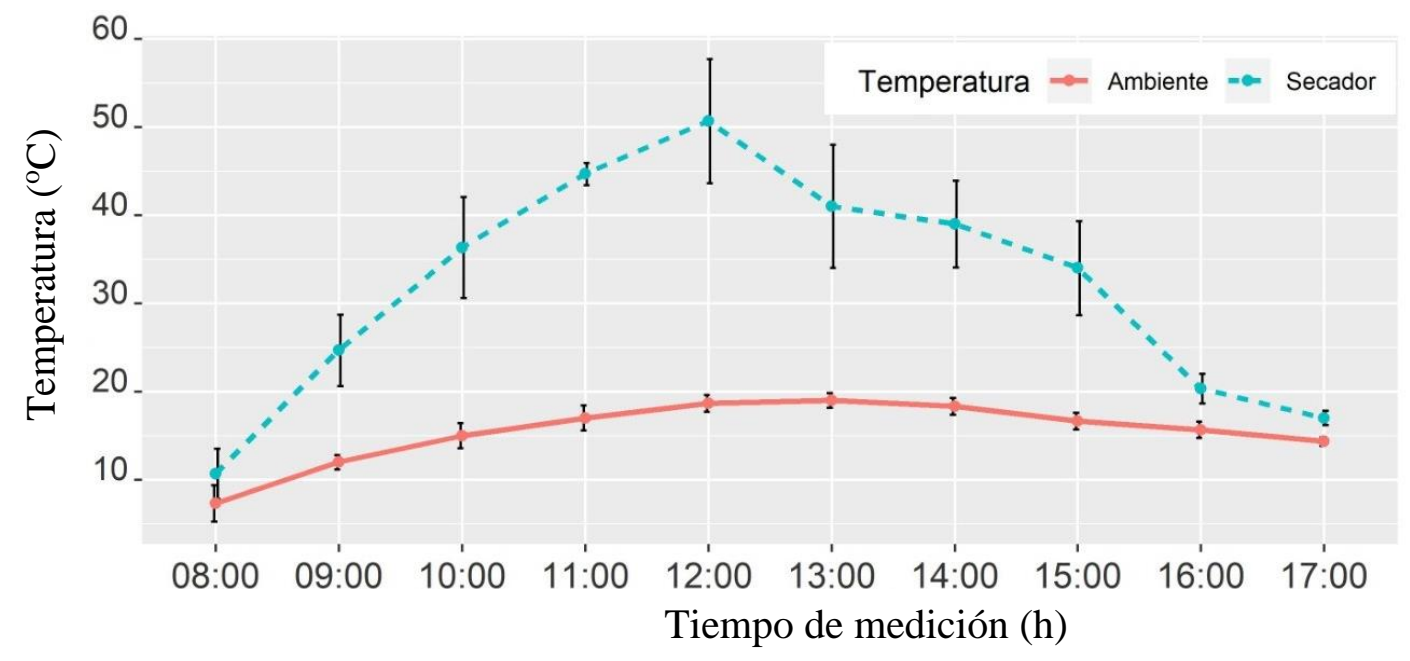

Figura 3. Temperaturas ambiente e interna del secador promedio, controlados a $55{ }^{\circ} \mathrm{C}$.

\section{Pruebas del secador solar automatizado con papa Yungay}

Para evaluar la viabilidad de secado de la papa Yungay en el secador solar automatizado, las papas fueron en primer lugar peladas y trozadas en forma de cubos de $1 \times 1.5 \mathrm{~cm}$, en total 50 cubos con un peso por muestra ensayada de $850 \mathrm{~g}$. Estos cubos fueron sumergidos durante 5 min a escaldado utilizando una solución del bisulfito de sodio a dos diferentes concentraciones: $0.02 \%$ y $0.03 \% \mathrm{p} / \mathrm{v}$, una vez retirados se escurrieron por 5 minutos y se procede a su secado a tres temperaturas: $50{ }^{\circ} \mathrm{C}$, $55{ }^{\circ} \mathrm{C}$ y $60{ }^{\circ} \mathrm{C}$ por un tiempo aproximado de $72 \mathrm{~h}, 3$ días consecutivos, obteniendo una reducción de $70 \%$ (de $850 \mathrm{~g}$ a $250 \mathrm{~g}$, de 67\% a 13\%) en el peso de la muestra. En el Cuadro 2, se muestran las características fisicoquímicas analizadas después del proceso de secado.

\section{Cuadro 2. Contenido físico, fisicoquímico y químico proximal de papa seca a tres temperaturas} de secado.

\begin{tabular}{ccccccc}
\hline $\begin{array}{c}\text { Temperatura } \\
\text { de secado }\end{array}$ & $\begin{array}{c}\text { Concentración } \\
\text { de sulfito } \\
\text { Media } \pm \text { DS }\end{array}$ & $\begin{array}{c}\text { Humedad }(\%) \\
\text { Media } \pm \text { DS }\end{array}$ & $\begin{array}{c}\text { Acidez } \\
\text { Media } \pm \text { DS }\end{array}$ & $\begin{array}{c}\text { Ceniza } \\
\text { Media } \pm \text { DS }\end{array}$ & $\begin{array}{c}\text { MH } \\
\text { Media } \pm D S\end{array}$ & $\begin{array}{c}\text { Luminosidad } \\
\left(L^{*}\right)\end{array}$ \\
\hline $50{ }^{\circ} \mathrm{C}$ & $0.02 \%$ & $13.48 \pm 0.06 \mathrm{a}$ & $0.22 \pm 0.03 \mathrm{a}$ & $2.44 \pm 0.02 \mathrm{a}$ & $6.23 \pm 0.01 \mathrm{a}$ & $58.4 \pm 0.36 \mathrm{a}$ \\
& $0.03 \%$ & $13.6 \pm 0.14 \mathrm{a}$ & $0.19 \pm 0.02 \mathrm{a}$ & $2.49 \pm 0.01 \mathrm{a}$ & $6.23 \pm 0.01 \mathrm{a}$ & $58.31 \pm 0.09 \mathrm{a}$ \\
$55{ }^{\circ} \mathrm{C}$ & $0.02 \%$ & $13.44 \pm 0.05 \mathrm{a}$ & $0.17 \pm 0.01 \mathrm{a}$ & $2.31 \pm 0.11 \mathrm{a}$ & $6.27 \pm 0.01 \mathrm{a}$ & $58.63 \pm 0.15 \mathrm{a}$ \\
& $0.03 \%$ & $13.45 \pm 0.03 \mathrm{a}$ & $0.15 \pm 0.02 \mathrm{a}$ & $2.32 \pm 0.05 \mathrm{a}$ & $6.29 \pm 0 \mathrm{a}$ & $58.62 \pm 0.07 \mathrm{a}$ \\
$60{ }^{\circ} \mathrm{C}$ & $0.02 \%$ & $12.55 \pm 0.02 \mathrm{a}$ & $0.13 \pm 0.01 \mathrm{a}$ & $2.43 \pm 0.04 \mathrm{a}$ & $6.32 \pm 0.01 \mathrm{a}$ & $58.65 \pm 0.02 \mathrm{a}$ \\
& $0.03 \%$ & $12.74 \pm 0.02 \mathrm{a}$ & $0.12 \pm 0.02 \mathrm{a}$ & $2.38 \pm 0.01 \mathrm{a}$ & $6.27 \pm 0.03 \mathrm{a}$ & $58.5 \pm 0.46 \mathrm{a}$ \\
\hline
\end{tabular}

Valores con diferentes letras dentro de cada columna denotan diferencia significante $(p<0.05)$, valores promedio de tres repeticiones \pm desviación estándar (DS). 
Del Cuadro 2, se nota que al evaluar la interacción del pretratamiento con bisulfito y las temperaturas de secado no mostraron diferencia significativa $(p>0.05)$ en sus características físicas, fisicoquímicas y químico proximal: humedad, acidez, ceniza, $\mathrm{pH}$ y luminosidad. Infiriendo que una temperatura de 50 y concentración de $0.02 \%$ de bisulfito de sodio serian óptimas para la obtención de la papa seca. A estas temperaturas de secado los parámetros de calidad medidos confirman que el producto final es apto; asimimo, al evaluar la apariencia final del producto con respecto al atributo color todas las muestras presentan un color amarillo claro + café homogéneo.

\section{Pruebas del secador solar automatizado con papa}

Para la validación del secador solar automatizado se hizo pruebas de pretratamiento de la papa pelada (cubos de papa) con bisulfito para obtener papa seca como lo presentado en el Cuadro 2. Al respecto, Ahmed et al. (2010) evaluaron el efecto de pelado, secado a temperaturas entre $55^{\circ} \mathrm{C}-65$ ${ }^{\circ} \mathrm{C}$ y un pretratamiento de sus propiedades fisicoquímicas y su calidad nutricional.

Ellos señalaron que el bisulfito inhibe el pardeamiento enzimático causando una reacción entre los iones sulfito y las quininas inhibiendo la actividad polifenoloxidasa, el agotamiento del oxígeno y ofrece estabilidad al color del producto final. Ellos concluyeron que un producto de mejor calidad fue obtenido cuando las muestras son pretratadas con sulfito antes del secado a cualquier temperatura. Así mismo, el secado en el secador automatizado preserva el color en su parámetro de luminancia $\left(\mathrm{L}^{*}\right)$ y esta variable permite evaluar la degradación del color de la papa a secar (Espinoza, 2016).

La NTP 011.119 'papa y sus derivados: papa, definiciones y requisitos', establece como parámetros de calidad en la papa deshidratada un máximo de $15 \%$ de humedad y $2.5 \%$ de ceniza (Indecopi, 2010). Del Cuadro 2, se observa un máximo de humedad de $1.89 \%(0.02 \%$ concentración de bisulfito) y ceniza de $2.49 \%$ ( $0.03 \%$ concentración de bisulfito). De ello se establece que la humedad y ceniza de la papa Yungay seca obtenida en el secador solar automatizado se encuentra dentro de los parámetros de calidad de la norma 011.119. Se afirma que utilizando el secador solar y controlando los parámetros se obtuvo un produ cto de buena calidad y nos permite pasar a un desarrollo de secador solar automatizado industrial.

Cheftel (2000) señaló que la disponibilidad del agua (como solvente) induce a reacciones y brinda condiciones favorables para el desarrollo de microorganismos, los cuales podrían deteriorar el producto. En este caso el bajo porcentaje de humedad (13.89\%) comparado a la norma peruana (15\%) garantiza que nuestro producto (papa seca de Yungay) tendrá una mayor vida útil, ya que la probabilidad de propagación de microorganismos es mínima.

Waterschoot et al. (2016) señalan que el contenido de cenizas está relacionado muchas veces con la presencia de minerales tales como fósforo, sodio, potasio, magnesio y calcio. En este trabajo no fueron medidos tales minerales, pero suponemos la presencia de ellos debido a que la papa es cultivada en suelos donde existe la presencia de estos y que muchas veces son absorbidos a través de las raíces. Un reporte con el contenido de diferentes minerales en diferentes variedades de papa es reportado por el Ministerio de Salud (MINSA, 2017). Peña (2017), por su parte comento que la variabilidad del contenido de ceniza depende básicamente de la madurez del producto y de su procedencia. Los valores de ceniza en diferentes tipos de papa (por ejemplo, helada amarga, amarilla, chuño, blanca, etc.) están en un rango de $1.1 \%$ a $3.7 \%$ (MINSA, 2017). 
Obregon y Repo (2013) reportaron valores de $\mathrm{pH}$ 6.9, 6.6, 6.3 y 6.7 para las variedades de papa peruanita, amarilla runtus, huayro y huamantanga, respectivamente. Estos valores están en concordancia con los en este trabajo (Cuadro 2). El pH tiene una correlación inversamente proporcional a los valores de acidez (Salazar et al., 2008). Este comportamiento se muestra en el Cuadro 2, donde a mayores concentraciones de $\mathrm{pH}$, menores concentraciones de acidez.

Este comportamiento es debido a que el contenido de ácidos orgánicos de las papas varía según su estado de madurez. Ojeda et al. (2010) evaluaron trece diferentes clones de papas bajo diferentes ambientes (áreas). Resultados mostraron diferencias en el número de tallos, materia seca y índice de área foliar. Asimismo, fueron detectados diferencias en sus variables físicas (gravedad específica, materia seca, y diámetros polar y ecuatorial) y químicas (acidez, pH, almidón y azúcares). Estos resultados concuerdan con los encontrados en este trabajo.

\section{Conclusiones}

Se diseñó un secador solar autónomo y automatizado para el secado de productos agrícolas, el cual cuenta con un colector solar con dos reflectores, cámara de secado (seca de forma indirecta el producto), un sistema fotovoltaico que permite funcionar los ventiladores y sistemas de control de temperatura y humedad monitoreados por medio de un display electrónico. Se estudió el comportamiento térmico del secador al vacío (sin carga), logrando incrementos de la temperatura requeridas en la cámara de secado con radiaciones solares globales en condiciones de sierra, además se comprobó que el sistema automatizado de control permite programar las temperaturas requeridas en el proceso de secado de un producto agropecuario en condiciones óptimas.

El pretratamiento con bisulfito y las temperaturas establecidas en el secado de la papa Yungay no mostraron diferencia significativa $(p>0.05)$ en las características físicas, fisicoquímicas y químico proximal, de secado en el secador solar automatizado, cumpliendo así los parámetros de calidad de la NTP.011.110: 'papa y sus derivados: papa, definiciones y requisitos', con lo queda demostrado la viabilidad del secado de papa en el secador solar autónomo y automatizado, proponiéndolo como alternativa ambientalmente sostenible para el secado de calidad y bajos costos con respecto a otras alternativas convencionales.

\section{Agradecimientos}

Esta investigación fue financiada con los Fondos del Canon Minero que administra la Universidad Nacional del Centro del Perú, a través del Vicerrectorado de Investigación e Instituto de Investigación de la Universidad Nacional del Centro del Perú.

\section{Literatura citada}

Agraria. 2019. El 12\% de la producción nacional de papa se comercializa en el mercado mayorista de Lima. Agencia Agraria de Notícias. https://agraria.pe/noticias/el-12-de-la-produccionnacional-de-papa-se-comercializa-en-e-19088.

Ahmed, M.; Akter, M. S. and Eun, J. B. 2010. Peeling, drying temperatures, and sulphite-treatment affect physicochemical properties and nutritional quality of sweet potato flour. Food Chem. 121(1):112-118. https://doi.org/10.1016/j.foodchem.2009.12.015. 
AOAC. 1996. Official Methods of analysis. https://www.aoac.org/.

AOAC. 2006. Official Methods of Analysis of AOAC International (18 ${ }^{\text {th }}$ (Ed.). Issue February. AOAC International. https://www.researchgate.net/publication/292783651_AOAC_ 2005\#fullTextFileContent.

Bergues, C.; Griñan, P. and Martínez, A. 2008. Algunos aspectos de los cambios tecnológicos en secadores solares cubanos: realidades y tendencias. Tecnología Química. 28(2):35-45. https://www.redalyc.org/articulo.oa?id=445543756005.

Camayo-Lapa, B. F.; Pomachagua-Paucar, J. E.; Massipe-Hernaández, J. R.; Quispe-Flores, M. O. and Torres-Ten, A. 2017. Validación y aplicación del modelo Bristow Campbell para estimar la radiación solar global de la región de Junin. Tecnología Química. 37(3):574-590. http://scielo.sld.cu/scielo.php?script=sci_arttext\&pid=S2224-61852017000300015.

Cheftel, J. C. 2000. Introducción a la bioquímica y tecnología de los alimentos (Acribia (Ed.). $2^{\text {nd }}$ (Ed.). https://www.editorialacribia.com/libro/introduccion-a-la-bioquimica-y-tecnologiade-los-alimentos-volumen-ii.53732/.

Chemkhi, S.; Zagrouba, F. and Bellagi, A. 2004. Drying of agricultural crops by solar energy. Desalination. 168(1-3):101-109. https://doi.org/10.1016/j.desal.2004.06.174.

Chouicha, S.; Boubekri, A.; Mennouche, D. and Berrbeuh, M. H. 2013. Solar drying of sliced potatoes. An experimental investigation. Energy Procedia. 36(1):1276-1285. https://doi.org/10.1016/j.egypro.2013.07.144.

Daut, I.; Yusoff, M. I.; Ibrahim, S.; Irwanto, M. and Gomesh, N. 2012. Relationship between the solar radiation and surface temperature in Perlis. Adv. Materials Res. 512(5):143-147. https://doi.org/10.4028/www.scientific.net/AMR.512-515.143.

El-Sebaii, A. A. and Shalaby, S. M. 2012. Solar drying of agricultural products: a review. Renewable Sustainable Energy Reviews. 16(1):37-43. https://doi.org/10.1016/j.rser. 2011.07.134.

Espinoza, J. 2016. Innovación en el deshidratado solar. Rev. Chilena Ing. 24(1):72-80. https://doi.org/10.4067/S0718-33052016000500010.

Iglesias, R.; José, R.; Lastres, O.; López, P.; Farrera, N. and Ibáñez, G. 2017. Diseño, construcción y evaluación de un secador solar para mango Ataulfo. Rev. Mex. Cienc. Agríc. 8(8):1719-1732.

Indecopi. 2010. Papa. Definiciones y requisitos. NTP 011.110 2010. https://es.scribd.com/doc/ 52597721/Norma-tecnica-peruana-Papa-y-derivados.

Infoagro. 2020. Guía para el desarrollo de proyectos de secado solar en comunidades rurales. Energía sin fronteras. https://infoagronomo.net/guia-para-proyectos-de-secado-solar-enareas-rurales/.

Lati, M.; Boughali, S.; Bouguettaia, H.; Mennouche, D.; Bechki, D.; Khemgani, M. and Mir, B. 2017. Effect of solar drying on the quality of potato. International Conference on Green Energy and Environmental Engineering. 5(1):1-4. https://www.researchgate.net/ publication/317540200_Effect_of_solar_drying_on_the_quality_of_potato\#: :text=The

effect of solar drying, $\% 2 \mathrm{C}$ Reducing sugars and $\mathrm{pH}$.\&text=At this temperature the measured,color\%2C reducing sugar and $\mathrm{pH}$.

López, I. C. y Chávez, E. G. 2018. Eficacia de secador solar tipo túnel con cacao (theobroma cacao L.) en Tabasco. Rev. Mex. Cienc. Agríc. 21(1):4395-4405. https://doi.org/ 10.29312/remexca.v0i21.1528.

MINSA. 2017. Tablas peruanas de composición de alimentos. In: Perú $10^{\text {th }}$ (Ed.). SEGEAR SAC. https://doi.org/http://www.ins.gob.pe/insvirtual/images/otrpubs/pdf/Tabla\%20de\%20Alim entos.pdf. 
Obregón, A. y Repo, R. 2013. Evaluación fisicoquímica y bromatológica de cuatro variedades nativas de papa (Solanum spp.). Ciencia e Investigación. 16(1):38-40. https://revistasinvestigacion.unmsm.edu.pe/index.php/farma/article/view/8634/7478.

Ojeda, M.; De Camacaro, M. P.; Rodríguez, D.; Gallardo, M. y Valera, R. 2010. Evaluación hortícola, producción y calidad postcosecha de clones avanzados de papa en la Localidad de duaca, estado lara, venezuela. Bioagro. 22(1):17-28. https://www.researchgate.net/ publication/237041468_Evaluacion_horticola_-produccion_y_calidad_postcosecha_de_ clones_avanzados_de_papa_en_la_localidad_de_Duaca_estado_Lara_Venezuela.

Peña, E. C. 2017. Extracción y caracterización fisicoquímica y funcional de almidones de cinco variedades de papas nativas procedentes de Ilave (Puno). Universidad Nacional Agraria la Molina. http://repositorio.lamolina.edu.pe/bitstream/handle/UNALM/2655/Q02-P45T.pdf? sequence $=1 \&$ is Allowed $=\mathrm{y}$.

Prada, Á.; Vela, C. P.; Bardález, G. and Saavedra, J. 2019. Effectiveness of a coffee drying process using solar dryers with a continuous air flow system powered by photovoltaic energy, in the San Martín region, Peru. Información Tecnológica. 30(6):85-92. https://doi.org/10.4067/S0718-07642019000600085.

Quintanar, J. O.; Fuentes, M. E. L. y Tamarit, J. C. U. 2019. Evaluación económica de un secador solar para madera. Rev. Mex. Cienc. Forest. 2(7):97-104. https://doi.org/10.29298/ rmcf.v2i7.561.

$\mathrm{R}$ Team Core. 2019. A language and environment for statistical computing. R Foundation for Statistical Computing, Vienna Austria (3.2.6). R Foundation for Statistical Computing. https://www.r-project.org/.

Rodríguez-Tapia, B.; Villanueva-Vega, E.; Partida-Castaleda, A. y Avitia-Carlos, P. 2017. Diseño de un control de temperatura con técnicas de control clásico, para optimizar el proceso de deshidratado en un deshidratador solar. Rev. Aplic. Científ. Téc. 3(10):37-46. https://ecorfan.org/spain/researchjournals/Aplicacion_Cientifica_y_Tecnica/vol3num10/

Revista_de_Aplicacion_Cientifica_y_Tecnica_V3_N10_5.pdf.

Salazar, M.; Judith, Z. y Valecillos, H. 2008. Evaluación del rendimiento y características de calidad de trece clones avanzados de papa (Solanum tuberosum L.). Agricultura Andiina, 14(1):101-117. http://www.revencyt.ula.ve/storage/repo/ArchivoDocumento/agri/n14/ articulo7.pdf.

Seveda, M. S. and Jhajharia, D. 2012. Design and performance evaluation of solar dryer for drying of large cardamom (Amomum subulatum). J. Renewable Sustainable Energy. 4(6):1-11. https://doi.org/10.1063/1.4769199.

Tefera, A.; Endalew, W. and Fikiru, B. 2013. Evaluation and demonstration of direct solar potato drier. Livestock Research for Rural Development. 25(12):1-15. http://www.lrrd.org/ $\operatorname{lrd} 25 / 12 /$ teff25207.htm.

Waterschoot, J.; Gomand, S. V. and Delcour, J. A. 2016. Impact of swelling power and granule size on pasting of blends of potato, waxy rice and maize starches. Food Hydrocolloids. 52(1):69-77. https://doi.org/10.1016/j.foodhyd.2015.06.012. 